S1 Table. The accession numbers of neuraminidase sequences were used in present study

\title{
Influenza A(H3N2)
}

Virus name

A/California/7/2004

A/Wisconsin/67/2005

A/Brisbane/10/2007

A/Perth/16/2009

A/Victoria/361/2011

A/Switzerland/9715293/2013

A/Moscow/10/99

A/Panama/2007/1999

A/Beijing/353/1989

A/Beijing/32/1992

A/England/42/1972

A/Fujian/411/2002

A/Hong Kong/1/1968

A/Sichuan/2/1987

A/Sydney/5/1997

A/Texas/1/1977

A/Victoria/3/1975

A/Wuhan/359/1995

A/Wellington/01/2004

A/Israel/1/11/2011

A/Norway/1330/2010

A/Stockholm/18/2011

A/England/259/2011

A/Texas/50/2012

A/Hong Kong/146/2013

A/Ireland/M28390/2013

A/Hong Kong/4801/2014

A/Samara/73/2013

A/Newcastle/22/2014

A/Madagascar/0648/2011

A/Serbia/71/2011

A/Alabama/05/2010

A/Iowa/19/2010

A/Minnesota/10/2012

A/Victoria/208/2009

A/Bangkok/1/1979 1979
Accession number

EPI367106

EPI160220

EPI165491

EPI182942

EPI353905

EPI540525

EPI103336

DQ487337

DQ508835

CY033608

CY113087

CY088485

CY112251

CY112398

CY112887

CY113263

CY113183

CY112823

CY012106

EPI319251

EPI302230

EPI318273

EPI346608

KC892237

EPI426062

EPI467995

EPI539577

EPI460559

EPI541460

EPI319277

EPI326116

KC535442

KC882859

KC892316

EPI232452

CY121002 
A/Bangkok/SI-MI01/2015

A/Bangkok/SI-MI02/2015

A/Bangkok/SI-MI03/2015

A/Bangkok/SI-MI04/2015

A/Bangkok/SI-MI05/2014

A/Bangkok/SI-MI06/2014

A/Bangkok/SI-MI07/2014

A/Bangkok/SI-MI08/2014

A/Bangkok/SI-MI09/2014

A/Bangkok/SI-MI10/2014

A/Bangkok/SI-MI11/2014

A/Bangkok/SI-MI12/2014

A/Bangkok/SI-MI13/2014

A/Bangkok/SI-MI14/2014

A/Bangkok/SI-MI15/2014

A/Bangkok/SI-MI16/2014

A/Bangkok/SI-MI17/2014

A/Bangkok/SI-MI18/2014

A/Bangkok/SI-MI19/2014

A/Bangkok/SI-MI20/2014

A/Bangkok/SI-MI21/2015

A/Bangkok/SI-MI22/2015

A/Bangkok/SI-MI23/2015

A/Bangkok/SI-MI24/2015

A/Bangkok/SI-MI25/2015

A/Bangkok/SI-MI26/2015

A/Bangkok/SI-MI27/2015

A/Bangkok/SI-MI28/2015

A/Bangkok/SI-MI29/2015

A/Bangkok/SI-MI30/2015

A/Bangkok/SI-MI31/2015

A/Bangkok/SI-MI32/2015

A/Thailand/173/2007

A/Thailand/308/2008

A/Thailand/315/2008

A/Thailand/322/2008

A/Thailand/340/2007

A/Thailand/380/2007

A/Thailand/381/2007

A/Thailand/429/2007

A/Thailand/447/2008
KP877373

KP877374

KP877375

KP877376

KP877377

KP877378

KP877379

KP877380

KP877381

KP877382

KP877383

KP877384

KP877385

KP877386

KP877387

KP877388

KP877389

KP877390

KP877391

KP877392

KP877393

KP877394

KP877395

KP877396

KP877397

KP877398

KP877399

KP877400

KP877401

KP877402

KP877403

KP877404

AB501496

AB501490

AB501497

AB501493

AB501499

AB501503

AB501504

AB501506

AB501491 
A/Thailand/461/2007

AB501507

A/Thailand/515/2008

AB501487

A/Thailand/536/2008

AB501498

A/Thailand/545/2008

AB501488

A/Thailand/764/2008

AB501508

A/Thailand/CU-1101/2008

EU625366

A/Thailand/CU-1102/2008

EU625367

A/Thailand/CU-1103/2008

EU625368

A/Thailand/CU-A114/2013

KP336092

A/Thailand/CU-A134/2013

KP336094

A/Thailand/CU-A153/2013

KP336095

A/Thailand/CU-A164/2013

KP336097

A/Thailand/CU-A166/2013

KP336096

A/Thailand/CU-A182/2013

KP336099

A/Thailand/CU-A196/2013

KP336098

A/Thailand/CU-A24/2013

KP336091

A/Thailand/CU-A305/2013

KP336103

A/Thailand/CU-A411/2013

KP336112

A/Thailand/CU-A459/2013

KP336114

A/Thailand/CU-A598/2014

KP336121

A/Thailand/CU-A7/2013

KP336090

A/Thailand/CU-B10282/2014

KP336134

A/Thailand/CU-B10283/2014

KP336133

A/Thailand/CU-B10345/2014

KP336136

A/Thailand/CU-B10421/2014

KP336137

A/Thailand/CU-B10422/2014

KP336138

A/Thailand/CU-B10509/2014

KP336139

A/Thailand/CU-B10520/2014

KP336141

A/Thailand/CU-B10521/2014

KP336140

A/Thailand/CU-B10557/2014

KP336142

A/Thailand/CU-B106/2009

GQ983550

A/Thailand/CU-B10755/2014

KP336144

A/Thailand/CU-B10792/2014

KP336145

A/Thailand/CU-B10828/2014

KP336146

A/Thailand/CU-B10952/2014

KP336147

A/Thailand/CU-B10975/2014

KP336148

A/Thailand/CU-B110/2009

GQ902811

A/Thailand/CU-B11055/2014

KP336149

A/Thailand/CU-B11065/2014

KP336150

A/Thailand/CU-B11201/2014

KP336151

A/Thailand/CU-B11202/2014

KP336152 
A/Thailand/CU-B11284/2014

A/Thailand/CU-B11351/2014

A/Thailand/CU-B11367/2014

A/Thailand/CU-B1672/2009

A/Thailand/CU-B1697/2009

A/Thailand/CU-B4/2009

A/Thailand/CU-B4730/2011

A/Thailand/CU-B4836/2011

A/Thailand/CU-B4844/2011

A/Thailand/CU-B4929/2011

A/Thailand/CU-B5021/2011

A/Thailand/CU-B5105/2011

A/Thailand/CU-B5436/2011

A/Thailand/CU-B5465/2011

A/Thailand/CU-B5541/2011

A/Thailand/CU-B5593/2011

A/Thailand/CU-B5614/2011

A/Thailand/CU-B5697/2011

A/Thailand/CU-B5735/2011

A/Thailand/CU-B5773/2011

A/Thailand/CU-B5848/2011

A/Thailand/CU-B5873/2011

A/Thailand/CU-B590/2009

A/Thailand/CU-B5900/2011

A/Thailand/CU-B5909/2011

A/Thailand/CU-B5928/2011

A/Thailand/CU-B6091/2012

A/Thailand/CU-B6251/2012

A/Thailand/CU-B6274/2012

A/Thailand/CU-B6309/2012

A/Thailand/CU-B657/2009

A/Thailand/CU-B6780/2012

A/Thailand/CU-B6936/2012

A/Thailand/CU-B7189/2012

A/Thailand/CU-B7235/2012

A/Thailand/CU-B7269/2012

A/Thailand/CU-B7367/2012

A/Thailand/CU-B7418/2013

A/Thailand/CU-B7483/2013

A/Thailand/CU-B7536/2013

A/Thailand/CU-B7585/2013
KP336154

KP336155

KP336156

GU271976

GU271984

GQ902795

KP336040

KP336044

KP336042

KP336045

KP336046

KP336047

KP336049

KP336050

KP336051

KP336053

KP336052

KP336054

KP336055

KP336056

KP336057

KP336058

GQ902819

KP336059

KP336060

KP336061

KP336064

KP336065

KP336066

KP336067

GQ902827

KP336068

KP336070

KP336074

KP336076

KP336077

KP336078

KP336079

KP336080

KP336081

KP336082 
A/Thailand/CU-B7596/2013

A/Thailand/CU-B7646/2013

A/Thailand/CU-B7755/2013

A/Thailand/CU-B7765/2013

A/Thailand/CU-B7853/2013

A/Thailand/CU-B7885/2013

A/Thailand/CU-B7937/2013

A/Thailand/CU-B7992/2013

A/Thailand/CU-B8121/2013

A/Thailand/CU-B8127/2013

A/Thailand/CU-B8134/2013

A/Thailand/CU-B8222/2013

A/Thailand/CU-B8235/2013

A/Thailand/CU-B8236/2013

A/Thailand/CU-B8364/2013

A/Thailand/CU-B8518/2013

A/Thailand/CU-B8525/2013

A/Thailand/CU-B8736/2013

A/Thailand/CU-B8745/2013

A/Thailand/CU-B8772/2013

A/Thailand/CU-B8849/2014

A/Thailand/CU-C2417/2011

A/Thailand/CU-C4087/2013

A/Thailand/CU-C4364/2013

A/Thailand/CU-C4406/2014

A/Thailand/CU-C4492/2014

A/Thailand/CU-C4507/2014

A/Thailand/CU-C4546/2014

A/Thailand/CU-C4655/2014

A/Thailand/CU-CB166/2014

A/Thailand/CU-H1071/2009

A/Thailand/CU-H1285/2010

A/Thailand/CU-H1443/2010

A/Thailand/CU-H16/2009

A/Thailand/CU-H1817/2010

A/Thailand/CU-H2973/2011

A/Thailand/CU-H2989/2011

A/Thailand/CU-H3020/2011

A/Thailand/CU-H3141/2012

A/Thailand/CU-H3368/2012

A/Thailand/CU-H3434/2012
KP336083

KP336084

KP336085

KP336086

KP336087

KP336088

KP336089

KР336093

KP336100

KP336101

KP336102

KP336104

KP336106

KP336107

KP336108

KP336109

KP336110

KP336115

KP336116

KP336118

KP336122

KP336062

KP336105

KP336120

KP336123

KP336124

KP336126

KP336127

KP336132

KP336131

CY074944

CY074952

CY074960

GU271992

CY074968

KP336041

KP336043

KP336048

KP336063

KP336069

KP336071 
A/Thailand/CU-H3435/2012

A/Thailand/CU-H3453/2012

A/Thailand/CU-H3490/2012

A/Thailand/CU-H3567/2013

A/Thailand/CU-H3574/2013

A/Thailand/CU-H3580/2013

A/Thailand/CU-H3584/2013

A/Thailand/CU-H3595/2014

A/Thailand/CU-H3611/2014

A/Thailand/CU-H3624/2014

A/Thailand/CU-H3626/2014

A/Thailand/CU-H3649/2014

A/Thailand/CU-H3656/2014

A/Thailand/CU-H3680/2014

A/Thailand/CU-124/2006

A/Thailand/CU-228/2006

A/Thailand/CU-231/2006

A/Thailand/CU-259/2006

A/Thailand/CU-260/2006

A/Thailand/CU-272/2007

A/Thailand/CU-280/2007

A/Thailand/CU-282/2007

A/Thailand/CU-356/2008

A/Thailand/CU-370/2008

A/Thailand/CU-379/2008

A/Thailand/CU-46/2006

A/Thailand/Siriraj/02/2003

A/Thailand/Siriraj/03/2004

A/Thailand/Siriraj/06/2002

A/Thailand/Siriraj/08/1998

A/Thailand/VIROAF1/2012

A/Thailand/VIROAF1CS/2012

A/Thailand/VIROAF2/2012

A/Thailand/VIROAF6/2012

A/Bangkok/122/94

A/Siriraj/ICRC/NKS/1/2011

A/Tak/150/2015

A/Chanthaburi/13/2015

A/Nonthaburi/4/2015

A/Chanthaburi/49/2015

A/Bangkok/44/2015
KP336072

KP336073

KP336075

KP336111

KP336113

KP336117

KP336119

KР336125

KP336128

KP336129

KP336130

KP336135

KP336143

KP336153

EU021285

EU021275

EU021283

EU021279

EU021281

EU021271

EU021273

EU021277

FJ912978

FJ912986

FJ912994

EU021269

JN617983

JN617986

JN617984

JN617985

KJ577151

KJ848676

KJ577159

KJ577191

U43426

EPI670324

EPI652271

EPI652262

EPI652256

EPI649730

EPI649660 
A/Chanthaburi/41/2015

A/Bangkok/43/2015

A/Chanthaburi/40/2015

A/Chanthaburi/46/2015

A/Nakhonratchaisma/35/2015

A/Prachuapkhirikhan/289/2015

A/Nonthaburi/345/2015

A/Nonthaburi/340/2015

A/Rayong/335/2015

A/Nonthaburi/333/2015

A/Nong Khai/218/2013

A/Phuket/186/2013

A/Chanthaburi/179/2013

A/Tak/166/2013

A/Chiang Rai/164/2013

A/Bangkok/126/2013

A/NongKhai/185/2014

A/Chanthaburi/2419/2014

A/Nonthaburi/2421/2014

A/Nonthaburi/01/2015

A/Chanthaburi/170/2013

A/Phuket/342/2013

A/Tak/1845/2014

A/Nonthaburi/302/2014

A/Chanthaburi/301/2014

A/CHIANGRAI/186/2014

A/SONGKHLA/3076/2013

A/NONTHABURI/3027/2013

A/CHIANGRAI/337/2013

A/NONGKHAI/321/2013

A/BANGKOK/3489/2012

A/Prachuapkhirikhan/137/2014

A/Prachuapkhirikhan/163/2014

A/SongKhla/117/2014

A/ChiangRai/114/2014

A/Phuket/186/2013

A/Kamphaengphet/175/2013

A/Nonthaburi/174/2013

A/PRACHUAPKHIRIKHAN/408/2012

A/PHUKET/407/2012

A/Nonthanuri/456/2012
EPI649642

EPI649619

EPI649556

EPI649548

EPI649505

EPI649489

EPI647891

EPI647883

EPI647875

EPI647859

EPI587902

EPI587901

EPI587900

EPI587899

EPI587897

EPI587896

EPI567266

EPI566016

EPI565992

EPI564286

EPI553214

EPI553203

EPI545580

EPI543716

EPI543707

EPI541336

EPI529586

EPI529570

EPI529567

EPI529559

EPI526660

EPI520396

EPI520358

EPI516707

EPI516704

EPI467241

EPI466932

EPI466923

EPI450270

EPI450267

EPI432651 
A/Nonthaburi/263/2013

A/Prachuapkhirikhan/328/2012

A/Chanthaburi/324/2012

A/NONTHABURI/42/2012

A/SONGKHLA/8/2012

A/ChiangRai/277/2011

A/Nonthaburi/279/2011

A/ChiangRai/277/2011

A/Chonburi/269/2011

A/NONTHABURI/110/2011

A/CHIANGRAI/108/2011

A/CHIANGRAI/21/2011

A/SURATTHANI/23/2011

A/Thailand/911/2010

A/NOTHABURI/488/2010

A/Nonthaburi/342/2010

A/SURATTHANI/269/2010

A/SURATTHANI/116/2010

A/Bangkok/561/2009

A/Thailand/419/2009

A/Thailand/18/2009

A/Thailand/0316/2009

A/Thailand/01/2009

A/Tak/527/2009

A/SuratThani/559/2009

A/NongKhai/560/2009

A/BANGKOK/132/2009

A/SURATTHANI/72/2009

A/Thailand/981/2008

A/Thailand/947/2008

A/Thailand/860/2008

A/Thailand/998/2008

A/Thailand/944/2008

A/Thailand/419/2008

A/Thailand/356/2008

A/Thailand/786/2007

A/Thailand/677/2007

A/Thailand/284/2007

A/Thailand/271/2007

A/Thailand/786/2007

A/Thailand/677/2007
EPI432622

EPI406056

EPI406047

EPI379390

EPI379384

EPI348477

EPI346457

EPI346454

EPI346451

EPI346319

EPI346316

EPI331593

EPI331542

EPI295270

EPI294227

EPI278795

EPI272024

EPI272018

EPI232664

EPI232622

EPI232619

EPI232616

EPI232613

EPI232607

EPI232601

EPI232584

EPI228245

EPI211733

EPI185822

EPI185821

EPI185819

EPI175304

EPI175299

EPI163138

EPI163136

EPI162314

EPI162302

EPI162299

EPI162296

EPI158244

EPI158232 
A/Thailand/284/2007

EPI158223

A/Thailand/271/2007

EPI158220

A/Thailand/359/2007

EPI155714

A/THAILAND/656/2006

EPI155273

A/THAILAND/650/2006

EPI155271

A/THAILAND/707/2006

EPI155260

A/THAILAND/697/2006

EPI155256

A/THAILAND/692/2006

EPI155254

A/THAILAND/625/2006

EPI155250

A/THAILAND/618/2006

EPI155248

A/THAILAND/559/2006

EPI155246

A/Thailand/553/2006

EPI155244

A/THAILAND/558/2006

EPI155242

A/THAILAND/475/2006

EPI155240

A/Thailand/CU-B11417/2014

KX151186

A/Thailand/CU-B11444/2014

KX151187

A/Thailand/CU-B11461/2015

KX151188

A/Thailand/CU-B11507/2015

KX151189

A/Thailand/CU-B11518/2915

KX151190

A/Thailand/CU-A1402/2015

KX151191

A/Thailand/CU-B11566/2015

KX151192

A/Thailand/CU-B11585/2015

KX151193

A/Thailand/CU-C5500/2015

KX151194

A/Thailand/CU-A1513/2015

KX151195

A/Thailand/CU-H3689/2015

KX151196

A/Thailand/CU-C5572/2015

KX151197

A/Thailand/CU-B11686/2015

KX151198

A/Thailand/CU-B11703/2015

KX151199

A/Thailand/CU-B11773/2015

KX151200

A/Thailand/CU-B11807/2015

KX151201

A/Thailand/CU-B11820/2015

KX151202

A/Thailand/CU-B11843/2015

KX151203

A/Thailand/CU-B11870/2015

KX151204

A/Thailand/CU-B11889/2015

KX151205

A/Thailand/CU-B11935/2015

KX151206

A/Thailand/CU-B11968/2015

KX151207

A/Thailand/CU-H3700/2015

KX151208

A/Thailand/CU-B12006/2015

KX151209

A/Thailand/CU-B12034/2015

KX151210

A/Thailand/CU-B12069/2015

KX151211

A/Thailand/CU-B12081/2015

KX151212 
A/Thailand/CU-B12139/2015

A/Thailand/CU-H3708/2015

A/Thailand/CU-C5942/2015

A/Thailand/CU-B12191/2015

A/Thailand/CU-B12415/2015

A/Thailand/CU-B12589/2015

A/Thailand/CU-B12644/2015

A/Thailand/CU-C6062/2015

A/Thailand/CU-B12788/2015

A/Thailand/CU-B12888/2015

A/Thailand/CU-B13425/2015

A/Thailand/CU-C6193/2015

A/Thailand/CU-C6246/2015

A/Thailand/CU-B13893/2015
KX151213

KX151214

KX151215

KX151216

KX151217

KX151218

KX151219

KX151220

KX151221

KX151222

KX151223

KX151224

KX151225

KX151226

\section{Influenza A(H1N1)}

\section{Virus name}

A/NewCaledonia/20/1999

A/Denmark/16/2004

A/England/493/2006

A/Denmark/49/2006

A/Denmark/50/2006

A/Norway/2289/2006

A/Thailand/CU41/2006

A/Solomon Islands/3/2006

A/StPetersburg/8/2006

A/Thailand/CU75/2006

A/Guangzhou/1561/2006

A/Guangzhou/483/2006

A/Guangzhou/555/2006

A/Cambodia/365/2007

A/Hawaii/38/2007

A/Oregon/07/2007

A/Hawaii/46/2007

A/California/06/2008

A/Gunma/07G002/2008

A/Denmark/47/2006

A/England/594/2006

A/Niigata/F95/2007

\section{Accession number}

CY033624

EU097726

FJ445052

EU097736

EU097737

EPI509388

EU021247

EPI509400

CY035128

EU021263

EU382993

EU382988

EU382990

EPI157505

EU516270

EU516285

EU779656

GQ475837

CY043411

EU097731

FJ445066

CY094798 
A/Brisbane/59/2007

A/Tottori/52/2008

A/Hawaii/17/2009

A/Bangkok/163/2000

A/Thailand/1035/2008

A/Thailand/309/2008

A/Thailand/328/2006

A/Thailand/331/2006

A/Thailand/332/2006

A/Thailand/332/2008

A/Thailand/334/2006

A/Thailand/336/2006

A/Thailand/337/2006

A/Thailand/344/2006

A/Thailand/348/2006

A/Thailand/364/2006

A/Thailand/385/2006

A/Thailand/386/2006

A/Thailand/387/2006

A/Thailand/388/2006

A/Thailand/390/2006

A/Thailand/391/2006

A/Thailand/392/2006

A/Thailand/393/2006

A/Thailand/395/2006

A/Thailand/396/2006

A/Thailand/397/2006

A/Thailand/398/2006

A/Thailand/399/2006

A/Thailand/400/2006

A/Thailand/401/2006

A/Thailand/402/2006

A/Thailand/403/2006

A/Thailand/404/2006

A/Thailand/423/2006

A/Thailand/424/2006

A/Thailand/425/2006

A/Thailand/469/2008

A/Thailand/496/2008

A/Thailand/500/2007

A/Thailand/501/2007
CY058489

CY075491

EPI189495

CY125110

GQ423407

AB501444

AB501445

AB501446

AB501448

AB501447

AB501449

AB501450

AB501451

AB501452

AB501453

AB501454

AB501455

AB501456

AB501457

AB501458

AB501459

AB501460

AB501461

AB501462

AB501463

AB501464

AB501465

AB501466

AB501467

AB501468

AB501469

AB501470

AB501471

AB501472

AB501473

AB501474

AB501475

AB501476

AB501477

AB501478

AB501479 
A/Thailand/530/2008

A/Thailand/56/2008

A/Thailand/669/2008

A/Thailand/708/2006

A/Thailand/72/2006

A/Thailand/76/2006

A/Thailand/778/2008

A/Thailand/829/2008

A/Thailand/839/2008

A/Thailand/CU32/2006

A/Thailand/CU41/2006

A/Thailand/CU44/2006

A/Thailand/CU51/2006

A/Thailand/CU53/2006

A/Thailand/CU57/2006

A/Thailand/CU67/2006

A/Thailand/CU68/2006

A/Thailand/CU75/2006

A/Thailand/CU88/2006

A/Thailand/SirirajRamaTT/2004

A/Thailand/CU-H223/2009

A/Thailand/CU-H565/2009

A/Thailand/Siriraj/3043/2009

A/Thailand/CU-B589/2009

A/Thailand/CU-B685/2009

A/Thailand/CU-B97/2009

A/Thailand/CU-H17/2009

A/Thailand/CU-B267/2009

A/Thailand/CU-B42/2009

A/Thailand/874/2008

A/Thailand/1026/2008

A/Tak/427/2009

A/CHANTHABURI/341/2009

A/SURAT THANI/337/2009

A/TAK/334/2009

A/Thailand/1037/2008

A/Thailand/381/2008

A/Thailand/380/2008

A/Thailand/375/2008

A/Thailand/358/2008

A/Thailand/777/2007
AB501480

AB501481

AB526260

EU879083

AB501482

AB501483

AB501484

AB501485

AB501486

EU021265

EU021247

EU021259

EU021255

EU021249

EU021257

EU021251

EU021261

EU021263

EU021253

N676133

GU183817

GU271968

JN676131

GU183801

GU183809

GU271952

GQ902835

GU271960

GQ902803

EPI232891

EPI232890

EPI232888

EPI228273

EPI228257

EPI 228255

EPI189736

EPI163216

EPI163061

EPI163059

EPI163057

EPI158238 
A/Thailand/767/2007

EPI158236

A/Thailand/711/2007

EPI158234

A/Thailand/1577/2007

EPI158217

A/Suphanburi/2131/2004

EPI158162

A/Suphanburi/2130/2004

EPI158161

A/Suphanburi/2129/2004

EPI158160

A/Sakaew/2132/2004

EPI158099

A/Ratchaburi/1847/2004

EPI158089

A/Prachinburi/1817/2004

EPI158082

A/Prachinburi/1749/2004

EPI158080

A/Nonthaburi/1739/2004

EPI158031

A/Nonthaburi/1738/2004

EPI158029

A/Bangkok/1876/2004

EPI157428

A/Bangkok/1822/2004

EPI157427

A/Bangkok/1759/2004

EPI157426

A/Bangkok/1757/2004

EPI157425

A/Bangkok/1755/2004

EPI157424

A/Bangkok/1752/2004

EPI157423

A/Bangkok/1742/2004

EPI157422

A/Bangkok/1741/2004

EPI157421

A/Bangkok/1724/2004

EPI157420

A/Bangkok/1699/2004

EPI157419

A/Bangkok/1544/2004

EPI157418

A/Bangkok/1507/2004

EPI157416

A/Bangkok/1498/2004

EPI157415

A/Bangkok/1468/2004

EPI157414

A/Bangkok/1467/2004

EPI157413

A/Bangkok/1410/2004

EPI157412

A/Bangkok/1406/2004

EPI157411

A/Ayutthaya/1720/2004

EPI157410

A/Ayatthaya/2111/2004

EPI157408

A/Thailand/689/2006

EPI155285

A/Thailand/677/2006

EPI155283

A/Thailand/580/2006

EPI155281

A/Thailand/676/2006

EPI155279

A/Thailand/672/2006

EPI155277

A/Thailand/674/2006

EPI155275

A/Thailand/717/2006

EPI155268

A/Thailand/718/2006

EPI155266 
Influenza A(H1N1)pdm09

Virus name

A/California/07/2009

A/CzechRepublic/32/2011

A/HongKong/3934/2011

A/Christchurch/16/2010

A/Astrakhan/1/2011

A/HongKong/5659/2012

A/SouthAfrica/3626/2013

A/Dakar/02/2014

A/Ghana/DARI/0095/2014

A/StPetersburg/100/2011

A/Thailand/CU-B4656/2011

A/Thailand/CU-B4662/2011

A/Thailand/CU-B4717/2011

A/Thailand/CU-B4773/2011

A/Thailand/CU-B5356/2011

A/Thailand/CU-B5515/2011

A/Thailand/CU-B6475/2012

A/Thailand/CU-B6609/2012

A/Thailand/CU-B6801/2012

A/Thailand/CU-B8091/2013

A/Thailand/CU-B8092/2013

A/Thailand/CU-B8250/2013

A/Thailand/CU-A338/2013

A/Thailand/CU-B8573/2013

A/Thailand/CU-B8665/2013

A/Thailand/CU-B8730/2013

A/Thailand/CU-B8906/2014

A/Thailand/CU-B8908/2014

A/Thailand/CU-B8981/2014

A/Thailand/CU-B8982/2014

A/Thailand/CU-B9024/2014

A/Thailand/CU-B9037/2014

A/Thailand/CU-B9221/2014

A/Thailand/CU-B9225/2014

A/Thailand/CU-B9433/2014

A/Thailand/CU-H3628/2014

A/Thailand/CU-H3632/2014

A/Thailand/CU-B10114/2014

A/Thailand/CU-C4666/2014

A/Thailand/CU-B10032/2014
Accession number

FJ984386

EPI319448

EPI326207

EPI280343

EPI319591

EPI382425

EPI466627

EPI539471

EPI541030

EPI316434

KX151227

KX151228

KX151229

KX151230

KX151231

KX151232

KX151233

KX151234

KX151235

KX151236

KX151237

KX151238

KX151239

KX151240

KX151241

KX151242

KX151243

KX151244

KX151245

KX151246

KX151247

KX151248

KX151249

KX151250

KX151251

KX151252

KX151253

KX151254

KX151255

KX151256 
A/Thailand/CU-B10033/2014

KX151257

A/Thailand/CU-B10044/2014

KX151258

A/Thailand/CU-B10126/2014

KX151259

A/Thailand/CU-B10172/2014

KX151260

A/Thailand/CU-B10174/2014

KX151261

A/Thailand/CU-B10180/2014

KX151262

A/Thailand/CU-A1058/2014

KX151263

A/Thailand/CU-B10185/2014

KX151264

A/Thailand/CU-B10207/2014

KX151265

A/Thailand/CU-C4844/2014

KX151266

A/Thailand/CU-A1070/2014

KX151267

A/Thailand/CU-A1105/2014

KX151268

A/Thailand/CU-B10405/2014

KX151269

A/Thailand/CU-B10578/2014

KX151270

A/Thailand/CU-B10652/2014

KX151271

A/Thailand/CU-B10658/2014

KX151272

A/Thailand/CU-B10888/2014

KX151273

A/Thailand/CU-B10909/2014

KX151274

A/Thailand/CU-H3658/2014

KX151275

A/Thailand/CU-C5062/2014

KX151276

A/Thailand/CU-A1205/2014

KX151277

A/Thailand/CU-B11070/2014

KX151278

A/Thailand/CU-C5149/2014

KX151279

A/Thailand/CU-C5169/2014

KX151280

A/Thailand/CU-A1282/2014

KX151281

A/Thailand/CU-B11233/2014

KX151282

A/Thailand/CU-B11291/2014

KX151283

KX151284

A/Thailand/CU-C5263/2014

KX151285

A/Thailand/CU-A1344/2014

KX151286

A/Thailand/CU-B11363/2014

KX151287

A/Thailand/CU-B11375/2014

A/Thailand/CU-B11422/2014

KX151288

KX151289

A/Thailand/CU-A1434/2015

KX151290

A/Thailand/CU-B11495/2015

KX151291

A/Thailand/CU-B11463/2015

KX151292

A/Thailand/CU-B11556/2015

KX151293

A/Thailand/CU-B11612/2015

KX151294

KX151295

A/Thailand/CU-B11669/2015

KX151296

A/Thailand/CU-C5474/2015

KX151297

A/Thailand/CU-B11672/2015

KX151298

A/Thailand/CU-B11699/2015

KX151299 
A/Thailand/CU-C5535/2015

A/Thailand/CU-B11797/2015

A/Thailand/CU-B11841/2015

A/Thailand/CU-B11844/2015

A/Thailand/CU-B11877/2015

A/Thailand/CU-C5736/2015

A/Thailand/CU-B11984/2015

A/Thailand/CU-C5866/2015

A/Thailand/CU-B12167/2015

A/Thailand/CU-B12418/2015

A/Thailand/CU-B12591/2015

A/Thailand/CU-B12604/2015

A/Thailand/CU-B12653/2015

A/Thailand/CU-B12974/2015

A/Thailand/CU-B13002/2015

A/Thailand/CU-B6372/2012

A/Thailand/CU-B9280/2014

A/Thailand/CU-B9297/2014

A/Thailand/CU-H3640/2014

A/Thailand/CU-B13202/2015

A/Thailand/CU-B13274/2015

A/Thailand/CU-B13508/2015

A/Thailand/CU-C6326/2015

A/Thailand/CU-B14522/2015

A/Thailand/CU-B14530/2015

A_Bangkok_INS3_681_2012

A_Bangkok_INS424_2010

A/Bangkok/INS425/2010

A/Bangkok/INS426/2010

A/Bangkok/INS427/2010

A/Bangkok/INS428/2010

A/Bangkok/INS477/2010

A/Bangkok/INS478/2010

A/Bangkok/INS479/2010

A/Bangkok/INS480/2010

A/Bangkok/INS481/2010

A/Bangkok/INS482/2010

A/Bangkok/INS483/2010

A/Bangkok/INS484/2010

A/Bangkok/INS485/2010

A/Bangkok/INS486/2010

A/Bangkok/INS487/2010

A/Bangkok/INS488/2010
KX151300

KX151301

KX151302

KX151303

KX151304

KX151305

KX151306

KX151307

KX151308

KX151309

KX151310

KX151311

KX151312

KX151313

KX151314

KX151315

KX151316

KX151317

KX151318

KX151319

KX151320

KX151321

KX151322

KX151323

KX151324

CY176748

CY071329

CY071337

CY071345

CY071353

CY071361

CY096292

CY096300

CY096308

CY096316

CY096324

CY096332

CY096340

CY098108

CY096348

CY098116

CY098124

CY098132 
A/Bangkok/INS489/2010

CY096356

A/Bangkok/INS490/2010

CY098140

A/Bangkok/INS491/2010

CY096364

A/Bangkok/INS492/2010

CY096372

A/Bangkok/INS493/2010

CY096380

A/Bangkok/INS494/2010

CY096388

A/Bangkok/INS495/2010

CY096396

A/Bangkok/INS497/2010

CY096404

A/Bangkok/INS498/2010

CY096412

A/Bangkok/INS499/2010

CY096420

A/Bangkok/INS500/2010

CY096428

A/Bangkok/INS501/2010

CY096436

A/Bangkok/INS502/2010

CY098148

A/Bangkok/INS503/2010

CY096444

A/Bangkok/INS504/2010

CY096452

A/Bangkok/INS505/2010

CY096460

A/Bangkok/INS506/2010

CY096468

A/Bangkok/INS507/2010

CY096476

A/Bangkok/INS508/2010

CY098156

A/Bangkok/INS509/2010

CY098164

A/Bangkok/INS510/2010

CY096484

A/Bangkok/INS511/2010

CY096492

A/Bangkok/INS512/2010

CY096500

A/Bangkok/INS513/2010

CY096508

A/Bangkok/INS514/2010

CY096515

A/Bangkok/INS516/2010

CY098555

A/Bangkok/INS517/2010

CY096523

A/Bangkok/INS518/2010

CY096531

A/Bangkok/INS519/2010

CY096539

A/Bangkok/INS520/2010

CY098565

A/Bangkok/INS580/2010

CY129760

A/Bangkok/INS581/2010

CY129768

A/Bangkok/INS582/2010

CY129776

A/Bangkok/INS583/2010

CY129784

A/Bangkok/INS584/2010

CY129792

A/Bangkok/INS587/2010

CY129816

A/Bangkok/SIMI501/2009

KM013714

A/Bangkok/SIMI502/2010

KM013715

A/Bangkok/SIMI503/2010

KM013716

A/Bangkok/SIMI504/2010

KM013717

A/Bangkok/SIMI505/2010

KM013718

A/Bangkok/SIMI506/2010

KM013719

A/Bangkok/SIMI507/2009

KM013720 
A/Bangkok/SIMI508/2010

A/Bangkok/SIMI509/2010

A/Bangkok/SIMI510/2010

A/Bangkok/SIMI511/2010

A/Khon Kaen/INS3_640/2010

A/Khon Kaen/INS3_647/2010

A/Khon Kaen/INS3_649/2012

A/Khon Kaen/INS440/2010

A/Khon Kaen/INS441/2010

A/Khon Kaen/INS443/2010

A/Khon Kaen/INS444/2010

A/Khon Kaen/INS445/2010

A/Khon Kaen/INS446/2010

A/Khon Kaen/INS447/2010

A/Khon Kaen/INS448/2010

A/Khon Kaen/INS449/2010

A/Khon Kaen/INS450/2010

A/Khon Kaen/INS451/2010

A/Khon Kaen/INS452/2010

A/Khon Kaen/INS453/2010

A/Khon Kaen/INS454/2010

A/Khon Kaen/INS455/2010

A/Khon Kaen/INS456/2010

A/Khon Kaen/INS457/2010

A/Khon Kaen/INS458/2010

A/Khon Kaen/INS459/2010

A/Khon Kaen/INS460/2010

A/Khon Kaen/INS461/2010

A/Khon Kaen/INS462/2010

A/Khon Kaen/INS521/2010

A/Khon Kaen/INS522/2010

A/Khon Kaen/INS523/2010

A/Khon Kaen/INS585/2010

A/Khon Kaen/INS586/2010

A/Khon Kaen/INS588/2010

A/Nonthaburi/102/2009

A/Thailand/104/2009

A/Thailand/C602/2010

A/Thailand/CU-B2357/2010

A/Thailand/CU-B5/2009

A/Thailand/CU-H1786/2010

A/Thailand/CU-H1821/2010

A/Thailand/CU-H2176/2010
KM013721

KM013722

KM013723

KM013724

CY176468

CY176524

CY176540

CY093058

CY096068

CY096076

CY098204

CY096084

CY096092

CY096100

CY093066

CY096108

CY096116

CY096124

CY096132

CY096140

CY096148

CY096156

CY093074

CY096164

CY096172

CY096180

CY096188

CY098100

CY096196

CY096547

CY096555

CY096563

CY129800

CY129808

CY129824

CY039988

GQ169381

CY080309

CY080301

GQ866953

CY080325

CY080341

CY088803 
A/Thailand/CU-H2283/2010

CY082965

A/Thailand/CU-H2358/2010

CY082966

A/Thailand/CU-H2389/2010

CY082967

A/Thailand/CU-H2417/2010

CY088832

A/Thailand/CU-H2543/2010

CY088840

A/Thailand/CU-FS1/2014

KU051433

A/Thailand/CU-FS2/2014

KU051441

A/Thailand/H1255/2010

CY080317

A/Thailand/H1818/2010

CY080333

A/Thailand/NHRC430218/2014

KJ889263

A/Thailand/SirirajICRC_BKK_1/2010

KF849751

A/Thailand/SirirajICRC_CBI_2/2009

KF849738

A/Thailand/SirirajICRC_CBI_3/2009

KF849739

A/Thailand/SirirajICRC_CBI_4/2009

KF849740

A/Thailand/SirirajICRC_CBI_7/2009

KF849741

A/Thailand/SirirajICRC_CBI_8/2009

KF849742

A/Thailand/SirirajICRC_CBI_9/2009

KF849743

A/Thailand/SirirajICRC_NMA_1/2011

KF849752

A/Thailand/SirirajICRC_NMA_11/2011

KF849761

A/Thailand/SirirajICRC_NMA_12/2011

KF849762

A/Thailand/SirirajICRC_NMA_13/2011

KF849763

A/Thailand/SirirajICRC_NMA_14/2011

KF849764

A/Thailand/SirirajICRC_NMA_15/2011

KF849765

A/Thailand/SirirajICRC_NMA_16/2011

KF849766

A/Thailand/SirirajICRC_NMA_2/2011

KF849753

A/Thailand/SirirajICRC_NMA_3/2011

KF849754

A/Thailand/SirirajICRC_NMA_4/2011

KF849755

A/Thailand/SirirajICRC_NMA_5/2011

KF849756

A/Thailand/SirirajICRC_NMA_6/2011

KF849757

A/Thailand/SirirajICRC_NMA_7/2011

KF849758

A/Thailand/SirirajICRC_NMA_8/2011

KF849759

A/Thailand/SirirajICRC_NMA_9/2011

KF849760

A/Thailand/SirirajICRC_NSN_1/2010

KF849749

A/Thailand/SirirajICRC_NSN_3/2010

KF849750

A/Thailand/SirirajICRC_SEA-001(34)/2010

KF849747

A/Thailand/SirirajICRC_SEA-002(33)/2010

KF849745

A/Thailand/SirirajICRC_SEA-002(34)/2010

KF849744

A/Thailand/SirirajICRC_SEA-003(33)/2010

KF849748

A/Thailand/SirirajICRC_SEA-003(34)/2010

KF849746

A/Thailand/CU-H2548/2010

CY089449

A/Thailand/CU-H2698/2010

CY089457

A/Thailand/CU-H2911/2011

CY089465

A/Thailand/CU-H567/2009

CY074992 
A/Thailand/CU-H572/2009

CY075000

A/Thailand/CU-H847/2009

CY075008

A/Thailand/CU-H88/2009

HM446344

A/Thailand/CU-H9/2009

GQ866961

A/Thailand/CU-H910/2009

CY075016

A/Thailand/CU-C1157/2010

CY081157

A/Thailand/CU-C161/2009

CY074976

A/Thailand/CU-H1222/2010

CY074984

A/Thailand/CU-B4148/2010

CY089431

A/Thailand/CU-B4339/2010

CY089439

A/Chanthaburi/38/2015

EPI643493

A/Nonthaburi/53/2015

EPI643329

A/Nonthaburi/52/2015

EPI 643283

A/Nonthaburi/59/2015

EPI 643259

A/Pathumthani/12/2015

EPI636129

A/Chanthaburi/11/2015

EPI636091

A/Nonthaburi/375/2014

EPI565031

A/Nonthaburi/2398/2014

EPI 564983

A/Nonthaburi/72/2013

EPI552434

A/Bangkok/374/2013

EPI552432

A/Song Khla/160/2013

EPI552429

A/Phuket/01/2013

EPI 552427

A/Phuket/316/2014

EPI541523

A/Bangkok/1792/2014

A/SUPHANBURI/234/2014

EPI541517

EPI540902

A/NONTHABURI/248/2014

EPI540897

A/Sukhothai/214/2014

EPI 531877

A/Chanthaburi/190/2014

EPI 531836

A/PRACHUAP KHIRI KHAN/332/2013

EPI 529418

A/Song Khla/139/2014

EPI524286

A/Chiang Rai/157/2013

EPI 466936

A/SONG KHLA/416/2012

EPI 450281

A/Surat Thani/16/2013

EPI 439227

A/Chiang Rai/312/2012

EPI407400

A/Phuket/294/2012

EPI 407397

A/Song Khla/3113/2012

EPI407361

A/Tak/327/2012

EPI406034

A/Chiang Rai/312/2012

EPI 406031

A/Ayutthaya/283/2012

EPI386005

A/Nonthaburi/74/2012

EPI379535

A/SONG KHLA/40/2012

EPI346512

A/Song Khla/270/2011

EPI332584

A/Nonthaburi/78/2011

EPI 331545 
A/SONG KHLA/34/2011

A/Thailand/742/2010

A/Thailand/594/2010

A/CHIANG RAI/226/2010

A/SAMUTPRAKAN/102/2010

A/Surat Thani/563/2009

A/Ayutthaya/568/2009

A/Thailand/2944/2009
EPI 295512

EPI 295494

EPI 276899

EPI271972

EPI 231481

EPI231343

EPI 221043

EPI179068

\section{Influenza B}

\section{Virus name}

B/Victoria/02/1987

B/Yamagata/16/1988

B/Yamanashi/166/1998

B/Lee/40 1940

B/Russia/1969

B/Shanghai/361/2002

B/Malaysia/2506/2004

B/Florida/4/2006 2006

B/Brisbane/60/2008

B/Wisconsin/01/2010

B/Jiangsu/10/2003

B/Johannesburg/3964/2012

B/Brisbane/33/2008

B/England/393/2008

B/Victoria/304/2006

B/Uruguay/12/2008

B/FujianGulou/1272/2008

B/Singapore/19/2009

B/Argentina/R158/2010

B/Harbin/7/1994

B/Sichuan/379/99

B/Bangladesh/3333/2007

B/Stockholm/12/2011

B/Bangkok/141/1994

B/Bangkok/153/1990

B/Bangkok/163/1990

B/Bangkok/34/1999

B/Bangkok/54/1999

\section{Accession number}

CY018759

CY018767

CY019533

DQ792899

EF626638

EF541477

CY040451

CY033878

CY115153

CY115185

CY033846

EPI406273

CY149983

EPI211560

AGX18593

EPI172515

EPI366582

EPI193057

EPI301333

CY040443

AJ784087

AFH58308

EPI340833

CY019677

CY019613

CY019621

AY139056

AY139055 


\begin{tabular}{|c|c|}
\hline B/Chantaburi/218/2003 & CY022223 \\
\hline B/Thailand/SirirajBKK1/2012 & KF492944 \\
\hline B/Thailand/SirirajBKK2/2012 & KF492945 \\
\hline B/Thailand/SirirajBKK3/2012 & KF492946 \\
\hline B/Thailand/SirirajFlu/0006/2012 & KF492952 \\
\hline B/Thailand/SirirajFlu/007/2012 & KF492953 \\
\hline B/Thailand/SirirajFlu/014/2012 & KF492960 \\
\hline B/Thailand/SirirajRMSC1/2010 & KF492967 \\
\hline B/Thailand/SirirajEA3604/2008 & KF492916 \\
\hline B/Thailand/SirirajSI1335/2010 & KF492921 \\
\hline B/Thailand/SirirajSI1648/2010 & KF492926 \\
\hline B/Thailand/SirirajSI1659/2010 & KF492927 \\
\hline B/Thailand/SirirajSI2117/2010 & KF492929 \\
\hline B/Thailand/SirirajSI2210/2010 & KF492930 \\
\hline B/Thailand/VIROAF3/2012 & KJ577167 \\
\hline B/Thailand/VIROAF3CS/2012 & KJ848692 \\
\hline B/Thailand/VIROAF4/2012 & KJ577175 \\
\hline B/Bangkok/42/2015 & EPI644134 \\
\hline B/Chanthaburi/315/2015 & EPI644118 \\
\hline B/Nonthaburi/1/2015 & EPI636474 \\
\hline B/Bankgok/22/2015 & EPI636306 \\
\hline B/Nonthaburi/373/2014 & EPI565142 \\
\hline B/Prachuap/374/2014 & EPI562680 \\
\hline B/Nonthaburi/359/2014 & EPI562662 \\
\hline B/SongKhla/322/2014 & EPI541764 \\
\hline B/Phuket/317/2014 & EPI541759 \\
\hline B/ChiangRai/318/2014 & EPI541756 \\
\hline B/NongKhai/321/2014 & EPI541703 \\
\hline B/CHIANG RAI/273/2014 & EPI541360 \\
\hline B/PHUKET/266/2014 & EPI541349 \\
\hline B/PRACHUAP/233/2014 & EPI541344 \\
\hline B/CHANTHABURI/3046/2013 & EPI529350 \\
\hline B/PHUKET/3073/2013 & EPI529344 \\
\hline B/TAK/338/2013 & EPI529336 \\
\hline B/Tak/145/2014 & EPI526088 \\
\hline B/NongKhai/176/2014 & EPI526058 \\
\hline B/Sakaeo/73/2014 & EPI520499 \\
\hline B/Bangkok/132/2014 & EPI520496 \\
\hline B/NongKhai/172/2013 & EPI465931 \\
\hline B/ChiangRai/169/2013 & EPI465927 \\
\hline B/SongKhla/1598/2013 & EPI465914 \\
\hline
\end{tabular}




\begin{tabular}{|c|c|}
\hline B/Phuket/151/2013 & EPI465899 \\
\hline B/PHUKET/423/2012 & EPI450355 \\
\hline B/NONGKAI/419/2012 & EPI450349 \\
\hline B/CHIANGRAI/3518/2012 & EPI450334 \\
\hline B/ChiangRai/13/2013 & EPI433135 \\
\hline B/ChiangRai/15/2013 & EPI433126 \\
\hline B/NongKhai/03/2013 & EPI431406 \\
\hline B/PRACHUAP/446/2012 & EPI417388 \\
\hline B/NONTHANURI/2465/2012 & EPI417373 \\
\hline B/NONGKHAI/46/2012 & EPI417370 \\
\hline B/NONGKAI/2433/2012 & EPI417368 \\
\hline B/CHANTHABURI/2460/2012 & EPI417350 \\
\hline B/BANGKOK/29/2012 & EPI417329 \\
\hline B/Prachuap/78/2012 & EPI408663 \\
\hline B/NongKhai/322/2012 & EPI406963 \\
\hline B/Bangkok/282/2012 & EPI406956 \\
\hline B/Chanthaburi/318/2012 & EPI406950 \\
\hline B/SongKhla/85/2012 & EPI387729 \\
\hline B/Phuket/86/2012 & EPI387716 \\
\hline B/SONGKHLA/24/2012 & EPI379573 \\
\hline B/NONTHABURI/45/2012 & EPI379552 \\
\hline B/CHIANGRAI/50/2012 & EPI379518 \\
\hline B/PHUKET/53/2012 & EPI379515 \\
\hline B/CHIANGRAI/22/2012 & EPI379369 \\
\hline B/NONTHABURI/54/2012 & EPI379361 \\
\hline B/NONGKHAI/39/2012 & EPI379358 \\
\hline B/CHANTHABURI/32/2012 & EPI379350 \\
\hline B/Phuket/77/2012 & EPI378209 \\
\hline B/NongKhai/67/2012 & EPI378206 \\
\hline B/Tak/57/2012 & EPI378203 \\
\hline B/ChiangRai/55/2012 & EPI378200 \\
\hline B/Bangkok/271/2011 & EPI346961 \\
\hline B/NONTHABURI/1766/2011 & EPI346107 \\
\hline B/NONGKHAI/112/2011 & EPI346104 \\
\hline B/SONGKHLA/43/2011 & EPI332672 \\
\hline B/NONGKHAI/40/2011 & EPI332669 \\
\hline B/SURATTHANI/29/2011 & EPI331536 \\
\hline B/Chanthaburi/74/2011 & EPI331117 \\
\hline B/Chanthaburi/73/2011 & EPI331114 \\
\hline B/Thailand/820/2010 & EPI294862 \\
\hline B/BANGKOK/495/2010 & EPI294188 \\
\hline
\end{tabular}




\begin{tabular}{|c|c|}
\hline B/ChiangRai/369/2010 & EPI278935 \\
\hline B/SONGKHLA/236/2010 & EPI272005 \\
\hline B/CHANTHABURI/218/2010 & EPI272003 \\
\hline B/SONGKHLA/164/2010 & EPI272001 \\
\hline B/NONGKHAI/332/2009 & EPI228261 \\
\hline B/CHANTHABURI/329/2009 & EPI228259 \\
\hline B/Thailand/1024/2008 & EPI176457 \\
\hline B/Thailand/987/2008 & EPI176455 \\
\hline B/Thailand/3622/2008 & EPI162318 \\
\hline B/Thailand/3365/2008 & EPI162316 \\
\hline B/Thailand/393/2008 & EPI161761 \\
\hline B/Thailand/374/2008 & EPI161759 \\
\hline B/Thailand/373/2008 & EPI161757 \\
\hline B/Thailand/94/2007 & EPI157243 \\
\hline B/Thailand/89/2007 & EPI157241 \\
\hline B/Thailand/869/2007 & EPI157239 \\
\hline B/Thailand/810/2007 & EPI157237 \\
\hline B/Thailand/81/2007 & EPI157235 \\
\hline B/Thailand/762/2007 & EPI157232 \\
\hline B/Thailand/264/2007 & EPI157228 \\
\hline B/Thailand/1406/2007 & EPI157226 \\
\hline B/Phitsanulok/2053/2004 & EPI157161 \\
\hline B/Thailand/416/2007 & EPI155718 \\
\hline B/Thailand/400/2007 & EPI155716 \\
\hline B/Thailand/CU-243/2006 & JX513136 \\
\hline B/Thailand/CU-364/2008 & JX513144 \\
\hline B/Thailand/CU-A585/2013 & KM100243 \\
\hline B/Thailand/CU-A605/2014 & KM100244 \\
\hline B/Thailand/CU-A615/2014 & KM100247 \\
\hline B/Thailand/CU-A626/2014 & KM100248 \\
\hline B/Thailand/CU-A645/2014 & KM100251 \\
\hline B/Thailand/CU-B2201/2010 & JX512976 \\
\hline B/Thailand/CU-B2271/2010 & JX512984 \\
\hline B/Thailand/CU-B2320/2010 & JX512992 \\
\hline B/Thailand/CU-B2372/2010 & JX513000 \\
\hline B/Thailand/CU-B2390/2010 & JX513008 \\
\hline B/Thailand/CU-B2432/2010 & JX513016 \\
\hline B/Thailand/CU-B2504/2010 & JX513024 \\
\hline B/Thailand/CU-B2660/2010 & JX513032 \\
\hline B/Thailand/CU-B3153/2010 & JX513040 \\
\hline B/Thailand/CU-B4504/2011 & JX513048 \\
\hline
\end{tabular}




\begin{tabular}{|c|c|}
\hline B/Thailand/CU-B4585/2011 & JX513056 \\
\hline B/Thailand/CU-B5522/2011 & JX513064 \\
\hline B/Thailand/CU-B5671/2011 & JX513072 \\
\hline B/Thailand/CU-B5734/2011 & JX513080 \\
\hline B/Thailand/CU-B5910/2011 & JX513088 \\
\hline B/Thailand/CU-B6078/2012 & JX513096 \\
\hline B/Thailand/CU-B6096/2012 & JX513104 \\
\hline B/Thailand/CU-B6148/2012 & KM100232 \\
\hline B/Thailand/CU-B6240/2012 & KM100233 \\
\hline B/Thailand/CU-B6257/2012 & KM100234 \\
\hline B/Thailand/CU-B6975/2012 & KM100237 \\
\hline B/Thailand/CU-B7215/2012 & KM100239 \\
\hline B/Thailand/CU-B7337/2012 & KM100241 \\
\hline B/Thailand/CU-B8813/2013 & KM100242 \\
\hline B/Thailand/CU-B8925/2014 & KM100245 \\
\hline B/Thailand/CU-B8999/2014 & KM100249 \\
\hline B/Thailand/CU-B9017/2014 & KM100250 \\
\hline B/Thailand/CU-B9034/2014 & KM100252 \\
\hline B/Thailand/CU-C1262/2010 & JX513112 \\
\hline B/Thailand/CU-C1451/2010 & JX513120 \\
\hline B/Thailand/CU-C1768/2011 & JX513128 \\
\hline B/Thailand/CU-C4555/2014 & KM100253 \\
\hline B/Thailand/CU-H1400/2010 & JX513152 \\
\hline B/Thailand/CU-H1896/2010 & JX513160 \\
\hline B/Thailand/CU-H2132/2010 & JX513168 \\
\hline B/Thailand/CU-H2584/2010 & JX513176 \\
\hline B/Thailand/CU-H2738/2010 & JX513184 \\
\hline B/Thailand/CU-H2933/2011 & JX513192 \\
\hline B/Thailand/CU-H3002/2011 & JX513200 \\
\hline B/Thailand/CU-H3052/2011 & JX513208 \\
\hline B/Thailand/CU-H3313/2012 & KM100235 \\
\hline B/Thailand/CU-H3349/2012 & KM100236 \\
\hline B/Thailand/CU-H3456/2012 & KM100238 \\
\hline B/Thailand/CU-H3496/2012 & KM100240 \\
\hline B/Thailand/CU-H3591/2014 & KM100246 \\
\hline B/Thailand/CU-H3620/2014 & KX151325 \\
\hline B/Thailand/CU-C4610/2014 & KX151326 \\
\hline B/Thailand/CU-B9455/2014 & KX151327 \\
\hline B/Thailand/CU-B9693/2014 & KX151328 \\
\hline B/Thailand/CU-B10007/2014 & KX151329 \\
\hline B/Thailand/CU-B10030/2014 & KX151330 \\
\hline
\end{tabular}




\begin{tabular}{|c|c|}
\hline B/Thailand/CU-A832/2014 & KX151331 \\
\hline B/Thailand/CU-B10125/2014 & KX151332 \\
\hline B/Thailand/CU-B10127/2014 & KX151333 \\
\hline B/Thailand/CU-C4799/2014 & KX151334 \\
\hline B/Thailand/CU-B10233/2014 & KX151335 \\
\hline B/Thailand/CU-B10235/2014 & KX151336 \\
\hline B/Thailand/CU-B10236/2014 & KX151337 \\
\hline B/Thailand/CU-B10303/2014 & KX151338 \\
\hline B/Thailand/CU-B10340/2014 & KX151339 \\
\hline B/Thailand/CU-B10376/2014 & KX151340 \\
\hline B/Thailand/CU-B10440/2014 & KX151341 \\
\hline B/Thailand/CU-B10508/2014 & KX151342 \\
\hline B/Thailand/CU-B10739/2014 & KX151343 \\
\hline B/Thailand/CU-B10747/2014 & KX151344 \\
\hline B/Thailand/CU-B10835/2014 & KX151345 \\
\hline B/Thailand/CU-B11066/2014 & KX151346 \\
\hline B/Thailand/CU-B11155/2014 & KX151347 \\
\hline B/Thailand/CU-B11168/2014 & KX151348 \\
\hline B/Thailand/CU-C5175/2014 & KX151349 \\
\hline B/Thailand/CU-B11219/2014 & KX151350 \\
\hline B/Thailand/CU-B11259/2014 & KX151351 \\
\hline B/Thailand/CU-B11295/2014 & KX151352 \\
\hline B/Thailand/CU-B11305/2014 & KX151353 \\
\hline B/Thailand/CU-B11371/2014 & KX151354 \\
\hline B/Thailand/CU-B11389/2014 & KX151355 \\
\hline B/Thailand/CU-B11391/2014 & KX151356 \\
\hline B/Thailand/CU-B11425/2014 & KX151357 \\
\hline B/Thailand/CU-B11464/2015 & KX151358 \\
\hline B/Thailand/CU-B11465/2015 & KX151359 \\
\hline B/Thailand/CU-B11472/2015 & KX151360 \\
\hline B/Thailand/CU-B11572/2015 & KX151361 \\
\hline B/Thailand/CU-B11595/2015 & KX151362 \\
\hline B/Thailand/CU-B11603/2015 & KX151363 \\
\hline B/Thailand/CU-B11609/2015 & KX151364 \\
\hline B/Thailand/CU-B11673/2015 & KX151365 \\
\hline B/Thailand/CU-B11681/2015 & KX151366 \\
\hline B/Thailand/CU-B11705/2015 & KX151367 \\
\hline B/Thailand/CU-B11719/2015 & KX151368 \\
\hline B/Thailand/CU-B11774/2015 & KX151369 \\
\hline B/Thailand/CU-B11776/2015 & KX151370 \\
\hline B/Thailand/CU-B11789/2015 & KX151371 \\
\hline
\end{tabular}




$\begin{array}{ll}\text { B/Thailand/CU-B11823/2015 } & \text { KX151372 } \\ \text { B/Thailand/CU-B11863/2015 } & \text { KX151373 } \\ \text { B/Thailand/CU-B11864/2015 } & \text { KX151374 } \\ \text { B/Thailand/CU-B11901/2015 } & \text { KX151375 } \\ \text { B/Thailand/CU-B11908/2015 } & \text { KX151376 } \\ \text { B/Thailand/CU-B12073/2015 } & \text { KX151377 } \\ \text { B/Thailand/CU-B12092/2015 } & \text { KX151378 } \\ \text { B/Thailand/CU-B12096/2015 } & \text { KX151379 } \\ \text { B/Thailand/CU-B12113/2015 } & \text { KX151380 } \\ \text { B/Thailand/CU-B12741/2015 } & \text { KX151381 } \\ \text { B/Thailand/CU-B12992/2015 } & \text { KX151382 } \\ \text { B/Thailand/CU-B12998/2015 } & \text { KX151383 } \\ \text { B/Thailand/CU-B13258/2015 } & \text { KX151384 } \\ \text { B/Thailand/CU-B13297/2015 } & \text { KX151385 } \\ \text { B/Thailand/CU-B13716/2015 } & \text { KX151386 } \\ \text { B/Thailand/CU-B13874/2015 } & \text { KX151387 } \\ \text { B/Thailand/CU-B13917/2015 } & \text { KX151388 } \\ \text { B/Thailand/CU-B14903/2015 } & \text { KX151389 } \\ \text { B/Thailand/CU-B14300/2015 } & \text { KX151390 } \\ \text { B/Thailand/CU-B14301/2015 } & \text { KX151391 } \\ \text { B/Thailand/CU-B14308/2015 } & \text { KX151392 } \\ \text { B/Thailand/CU-B14349/2015 } & \text { KX151393 }\end{array}$

